\title{
An Updated Checklist of Bird Species in the Arjin Mountain Nature Reserve, China: Conservation Implications
}

\author{
Mardan Aghabey Turghan', Roller MaMing ${ }^{1 *}$, Li Weidong², Di Jie ${ }^{1}$ \\ and Xu Guohua ${ }^{1}$
}

${ }^{1}$ State Key Laboratory of Desert and Oasis Ecology, Xinjiang Institute of Ecology and Geography, Chinese Academy of Sciences, Urumqi, China, 830011

${ }^{2} \mathrm{Li}$ weidong Conservation Studio of Natural Ecology, Urumqi, China, 830011

\begin{abstract}
A B S T R A C T
An updated checklist of bird species that occur in the Arjin Mountain Nature Reserve and its adjacent areas, including parts of Kunlun Mountain, Qimantagh and Kumkul Basin, is provided as part of the basic data for a second nation-wide field survey of wildlife resources of China (2010-2020). The information provided is based on field observations made from 2010 to 2017. A total of 172 bird species belonging to 95 genera of 42 families of 19 orders were identified as occurring in the reserve, accounting for $37.9 \%$ of the total bird species in Xinjiang Uyghur Autonomous Region. Among them, 85 species are migrants, 49 species resident, 33 species summer visitors and 5 species winter visitors. Six species of birds viz., Redrumped Swallow (Cecropis daurica), Eastren Crowned Warbler (Phylloscopus coronatus), Blue-cheeked Bee-eater (Merops persicus), Robin Accentor (Prunella rubeculoides), Tibetan Rosefinch (Carpodacus roborowskii) and Japanese Sparrowhawk (Accipiter gularis) are being reported for the first time from Xinjiang. Among them, Blue-cheeked Bee-eater is reported from China for the first time.
\end{abstract}

\begin{tabular}{l} 
Article Information \\
Received 10 October 2018 \\
Revised 15 May 2019 \\
Accepted 22 October 2019 \\
Available online 12 February 2021 \\
Authors' Contribution \\
\hline MAT executed the project. RM \\
supervised of the project. LW was \\
responsible for the correct taxonomy \\
of species. DJ monitored bird species. \\
XG did field observation and data \\
analysis \\
Key words \\
Bird species, Diversity, Arjin \\
Mountain, Nature Reserve, \\
Conservation
\end{tabular}

\section{INTRODUCTION}

$\mathrm{T}$ The Arjin Mountain Nature Reserve (Fig. 1), part of one of the last great expanses of wilderness left on Earth, is located in Qarkilik County, Xinjiang Uyghur Autonomous Region, in western China (E87 $10^{\prime} \sim 91^{\circ} 18^{\prime}$, $\mathrm{N} 36^{\circ} 00^{\prime} \sim 37^{\circ} 49$ ) (Mardan et al., 2013). It was established in 1983 in order to protect its mountain ecosystem and was later upgraded to a National Nature Reserve in 1986 (Ablimit, 2004). Bordering the Qinghai-Tibetan Plateau to the north and covering an area of $45,000 \mathrm{~km}^{2}$, it is the second largest Nature Reserve in China after the Chang Tang Nature Reserve in Tibet, with which it is contiguous along its southern boundary (Ablimit, 2004; Ma et al., 2005). The altitude ranges from $3876 \mathrm{~m}$ at Ayak Kum to over $6973 \mathrm{~m}$ at Mount Muztagh (Chen, 1985). The entire area remains under snow cover from November to March. Broad, rolling alpine steppes broken by hills, glaciercapped mountains and large basins studded with wetlands and salt lakes are the visible forms of landscape in the region. The climate is characterized by dry, cold winters, strong winds, high levels of solar radiation, a wide range

\footnotetext{
* Corresponding author: maming @ms.xjb.ac.cn 0030-9923/2021/0002-0581 \$ 9.00/0

Copyright 2021 Zoological Society of Pakistan
}

of temperature between day and night, a short frost-free period, low precipitation and high evaporation. Annual precipitation varies from $200 \pm 300 \mathrm{~mm}$ while the annual evapotranspiration is about seven times greater. Precipitation is mainly concentrated in July and August. Annual temperature ranges from $-31{ }^{\circ} \mathrm{C}$ in January to $28{ }^{\circ} \mathrm{C}$ in July; the recorded lowest temperature is $-41^{\circ} \mathrm{C}$ (Mardan et al., 2013).

The remote and largely uninhabited reserve, characterized by high elevations, low annual precipitation, low nutrient levels and extremely cold weather in the winter, provides protection for a unique assemblage of wildlife (Schaller, 1998). The bird community inhabiting the reserve is unusual and diverse due to their geographic position as a part of the Tibetan Plateau and being situated at the junction of high mountains in the south and the Taklimakan Basin in the north. These varied habitats in terms of altitudinal and precipitation ranges and other environmental and topographic features, create an ideal setting for the high diversity of avifauna.

Bird species are an important indicator in biodiversity monitoring, and countries in Europe and North America have already been carrying out systematic surveys and monitoring of wild birds for more than 100 years, and bird diversity indices have become an official biodiversity monitoring tool in some of these countries $(\mathrm{Cu}$ et al., 
2013). However, China's Xinjiang province has not yet developed any formalized wild bird monitoring program, except for a few regional monitoring projects set up in the past decades. As for the research area, a broad view of the terrestrial vertebrates of the reserve has been given by previous researchers; however, few formal and specific scientific studies on bird species of the reserve are available (Qian et al., 1965; Zheng, 1976; Zhou and Cheng, 1985; Butler and Achuff, 1986; Gao, 1987; Achuff and Petocz, 1988; Huang and Gao, 1989; Feng, 1991; Ma et al., 2010). Furthermore, the main emphasis has been given to the eastern part of the reserve, which is relatively accessible, and it was roughly estimated that the reserve harbors 90 bird species belonging to 14 orders and 27 families (Gao, 1987). Because the current status and trends of bird species in the reserve are inadequately recorded or researched, it is difficult to come up with one set of data that may apply to all the bird species and their range, and, it must be stressed, that these figures come from several surveys conducted using different methods over a period of more than 20 years with the main focus on rare and endangered species, which are not representative of the overall status of wild birds, and have paid less attention to common wild birds. This situation called for a comprehensive survey of bird species of the reserve to better understand the species composition and their habitat associations so that a comprehensive management plan could be prepared for the conservation of this vital component of China's bird species biodiversity. For this reason, study on their current status is urgently required. The purpose of this article is to evaluate the current status of bird species in the Arjin Mountain Nature Reserve, as well as to document the major threats to their conservation.

\section{MATERIALS AND METHODS}

Fifteen field surveys were conducted, spanning a period of seven years, during spring and autumn respectively (2010-2017), at various localities that include selected representative habitats of Arjin Mountain Nature Reserve and its surrounding areas (Table I). The study area was divided into seven sites (Fig. 1) viz., 1. Atkhan (asbestos mines/settlements), 2. Kara Qokka (grazing areas/denser settlement), 3. Kara Dong (desert/ sparse vegetation/iron mines/scattered settlement), 4. Ixak Patti (wetland/scattered settlement), 5. Ayak Kum (desert steppe/sand hills/lake/no settlement), 6. Aqqik Kul (higher altitude above $4000 \mathrm{~m}$, alpine meadows/scattered settlement) and 7. Whale Lake (higher altitude above $5000 \mathrm{~m}$, altitude/ridges/alpine meadows/no settlement), which permitted complete coverage of the reserve. Each site represents a pre-existing administrative unit used as a protection station.

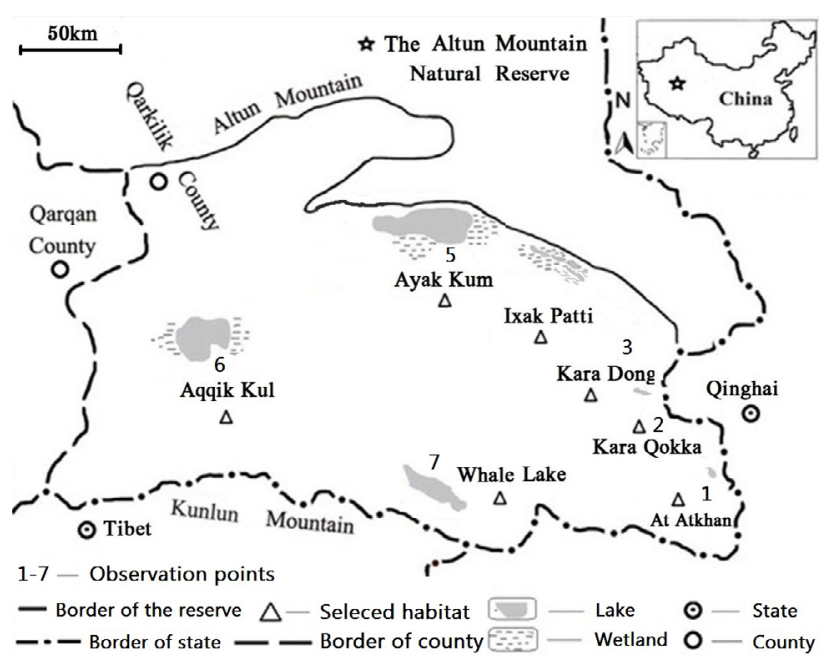

Fig. 1. The study sites of birds in the Altun Mountain Natural Reserve.

Seven observation points were chosen based on the representative habitats (At Atkhan $\rightarrow$ Kara Qokka $\rightarrow$ Kara Dong $\rightarrow$ Ixak $\quad$ Patti $\rightarrow$ Ayak $\quad \mathrm{Kum} \rightarrow$ Aqqik $\quad$ Kul $\rightarrow$ Whale Lake). Point surveys were undertaken employing visual observation method (Mardan et al., 2013) at each location by a pair of observers between 07:00 and 11:30 hour when the birds were most active. Birds were counted at each observation point of each location (Fig. 1), and recounted on a second visit on different days. This census period was seen as appropriate, because during the practice surveys, bird activity tended to be high during the whole morning. Birds were recorded at each census station for a period of 10 minutes. The distance from the recorder to each bird encountered by sight or by sound was estimated. The number of individuals in each encounter was recorded, if ascertainable. When approaching a census site, if any birds were disturbed (flushed) from the plot, these were recorded as being present during the census period. The census period commenced immediately on arrival at the sites. To avoid repeat counts of the same individuals, disturbing the birds was avoided as far as possible, and the direction of the movements of birds was carefully observed. Data recorded included date, time, GPS location, groups and population size, vegetation type, water sources, grassland fences, mining sites, roads and domestic sheep as well as signs of birds such as foot prints, feather, nests, feces, eggs etc.

Secondly, interviews with local wardens and pastoralists, and officials of the administrative bureau of the reserve, were administered to get information on the long-term population dynamics of avifauna of the reserve. 
The severities the potential threats to the avifauna at different sites were estimated based on literature reviews, preliminary interviews and field assessment (Mardan et al., 2011).

\section{RESULTS AND DISCUSSION}

Bird species were distributed in all the survey sites in the Arjin Mountain Nature Reserve.

\section{Population status}

A total of 172 bird species belonging to 95 genera of 42 families of 19 orders were identified as occurring in the reserve and its surrounding areas, accounting for $37.9 \%$ of the total bird species reported in Xinjiang Uyghur Autonomous Region (Xinjiang).

Six species of birds viz., Japanese sparrowhawk (Accipiter gularis), Eastern crowned warbler (Phylloscopus coronatus), blue-cheeked bee-eater (Merops persicus), robin accentor (Prunella rubeculoides) and tibetan rosefinch (Carpodacus roborowskii) are being reported for the first time from Xinjiang. Among them, blue-cheeked bee-eater is reported for the first time in China. In comparison to previous reports, more than 80 species belonging to 11 families of three orders are newly added in our updated checklist, while seven species, ring-necked (common) pheasant (Phasianus colchicus), white-backed woodpecker (Dendrocopos leucotos), Xinjiang ground-jay (Podoces biddulphi), white-browed bush-dweller (Rhopophilus pekinensis), saxaul sparrow (Passer ammodendri), desert finch (Rhodospiza obsoleta) and tawny-headed mountain finch (Leucosticte sillemi) are removed from the checklist based on the fact that their known range does not overlap with the territory of the reserve (Gao, 1987).

The abundance of avifauna of the reserve was deduced from the relative proportions of the number of individuals of the species, which were recorded in each encounter at all 15x7 sampling events (Table II). However, not all points were sampled at all time periods (Table I). In addition, the sampling and statistical methods employed were not robust, suggesting that our result on the abundance of avifauna of the reserve may not quite accurate. Further research with more robust sampling method including randomly located sample points was recommended to generate a more scientifically conducted population estimate.

The Passeriformes, which comprised 80 of the species accounted for $46.5 \%$ of the total bird species richness in the reserve. The Charadriiformes had the second highest species richness with 22 species $(12.8 \%)$, followed by Falconiformes with 19 species (11\%), Anseriformes with 17 species $(9.9 \%)$, and the remaining 34 species (19.8\%) are represented by other 15 orders.

The birds living in residential areas have a tendency of declining in recent years, implying that threats such as urbanization, over-grazing and illegal access into the reserve has not been effectively under control.

\section{Distribution pattern}

The study area falls between Tibetan and Xinjiang bio-regions. Consequently, the distribution pattern of its avifauna is characterized by inter-permeation between Tibetan Pattern (27 species, 15.7\%) and Central Asian pattern (49 species, 28.5\%). About 71 (41.3\%) species belong to the Northern pattern, including 52 species of Palaearctic (Ancient Northern) pattern, 17 species of Holarctic pattern and 2 species of Northeastern pattern. Most of the Northern pattern species are migrants, which account for the highest species richness and highest bird abundance of the reserve. Forty nine (28.5\%) species belong to the Central-Asian pattern. Twenty seven $(15.7 \%)$ species belong to the plateau (Highland) pattern, including the endemic species of the Tibetan Plateau such as black-necked crane (Grus nigricollis), Tibetan snowcock (Tetraogallus tibetanus), bar-headed goose (Anser indicus), brown-headed gull (Larus brunnicephalus), and Montifringilla species, and in spite of their relatively lower species richness, the highland birds account for considerably higher bird abundance, being the dominant avifauna throughout the reserve. Twenty three species $(13.4 \%)$ belong to the Circumboreal pattern. Long-tailed shrike (Lanius schach), which is widely distributed in China, was recorded as the single species belonging to Oriental pattern.

\section{Residence types and conservation status}

Among the 172 species recorded, 85 species are migrants (49.4\%), 49 species residents (28.4\%), 33 species summer visitors $(19.2 \%)$ and 5 species winter visitors $(2.9 \%)$. According to their residence types; the number of migrants is the largest, followed by residents and summer visitors, while the number of winter migrant birds is the least. There is a variety of rare and endangered bird species inhabiting the reserve. Among them, 6 and 23 species were listed respectively as category I and II Key National Protected Species under the Chinese Wild Animal Protection Law (Jiang, 2020). Two, 6, 7 and 2 species are listed as globally Endangered, Vulnerable, Near Threatened and Data Deficient respectively on the IUCN Red list (https://www.iucnredlist.org/). 
Table I. Dates of surveys of birds in Arjin Mountain Nature Reserve on the basis of their preferred habitats (2010-2017).

\begin{tabular}{|c|c|c|c|c|c|c|c|c|}
\hline Year & Date of survey & At Atkhan & Kara Qokka & Kara Dong & Ixak Patti & Ayak Kum & Aqqik Kul & Whale Lake \\
\hline Spr. 2010 & May8-25 & + & + & + & + & + & + & - \\
\hline Aut.2010 & Sept 13-Sept29 & + & + & + & + & + & + & + \\
\hline Spr. 2011 & May24-June 13 & + & + & + & + & + & + & + \\
\hline Aut.2011 & Aug28-Sept14 & + & + & + & + & + & + & + \\
\hline Spr. 2012 & May11-28 & + & + & + & + & + & + & - \\
\hline Aut.2012 & Oct6-23 & + & + & + & + & + & + & - \\
\hline Aut.2013 & Sept 06-22 & + & + & + & + & + & + & + \\
\hline Spr. 2014 & May16-June 6 & + & + & + & + & + & + & - \\
\hline Aut.2014 & Oct11-28 & + & + & + & + & + & + & - \\
\hline Spr. 2015 & May 05-23 & + & + & + & + & + & - & - \\
\hline Aut.2015 & Sept 01-21 & + & + & + & + & + & + & + \\
\hline Spr. 2016 & May15-June 2 & + & + & + & + & + & + & - \\
\hline Aut.2016 & Sept 1-Sept 20 & + & + & + & + & + & + & + \\
\hline Spr. 2017 & June $2-20$ & + & + & + & + & + & + & + \\
\hline
\end{tabular}

+ , Surveyed; -, Not surveyed; mainly for the extreme weather conditions and high altitude.

Table II. Distribution of birds in Arjin Mountain Nature Reserve on the basis of their preferred habitats.

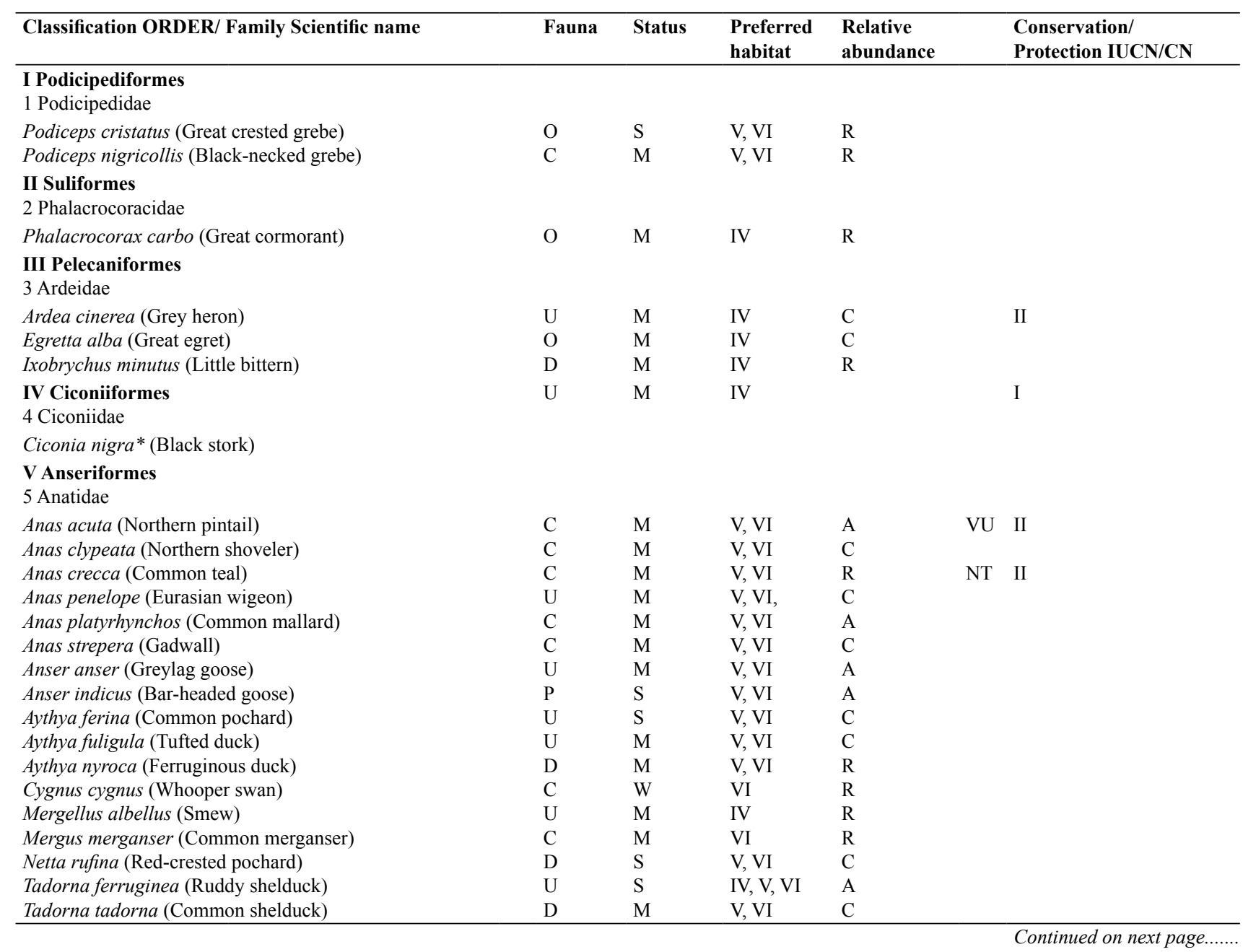




\begin{tabular}{|c|c|c|c|c|c|c|}
\hline Classification ORDER/ Family scientific name & Fauna & Status & $\begin{array}{l}\text { Preferred } \\
\text { habitat }\end{array}$ & $\begin{array}{l}\text { Relative } \\
\text { abundance }\end{array}$ & & $\begin{array}{l}\text { Conservation/ } \\
\text { Protection IUCN/CN }\end{array}$ \\
\hline \multicolumn{7}{|l|}{ VI Falconiformes } \\
\hline Accipiter gularis (Japanese sparrowhawk) & U & M & III, VI, VII & $\mathrm{R}$ & NT & II \\
\hline Accipiter nisus (Eurasian sparrowhawk) & $\mathrm{U}$ & M & II, III & $\mathrm{R}$ & & II \\
\hline Aegypius monachus (Cinereous vulture) & $\mathrm{D}$ & $\mathrm{R}$ & III, VI, VII & $\mathrm{R}$ & VU & II \\
\hline Aquila chrysaetos (Golden eagle) & $\mathrm{C}$ & $\mathrm{R}$ & III, VI, VII & $\mathrm{R}$ & VU & I \\
\hline Aquila heliaca (Eastern imperial eagle) & $\mathrm{D}$ & M & III, VI, VII & $\mathrm{R}$ & & II \\
\hline Aquila rapax (Steppe eagle) & $\mathrm{D}$ & M & III, VI, VII & $\mathrm{R}$ & & I \\
\hline Buteo hemilasius (Upland buzzard) & $\mathrm{D}$ & $\mathrm{R}$ & III, VI, VII, & $\mathrm{R}$ & & II \\
\hline Buteo lagopus (Rough-legged buzzard) & $\mathrm{C}$ & M & III, VI, VII & $\mathrm{R}$ & & I \\
\hline Buteo rufinus (Long-legged buzzard) & $\mathrm{D}$ & M & III, VI, VII & $\mathrm{R}$ & & II \\
\hline Circus macrourus* (Pallid harrier) & $\mathrm{D}$ & $\mathrm{R}$ & III, VI, VII & & NT & II \\
\hline Gypaetus barbatus (Bearded vulture) & $\mathrm{D}$ & $\mathrm{R}$ & III, VI, VII & $\mathrm{R}$ & & II \\
\hline Gyps himalayensis (Himalayan vulture) & $\mathrm{P}$ & $\mathrm{R}$ & III, VI, VII & $\mathrm{R}$ & EN & I \\
\hline Haliaeetus leucoryphus (Pallas's fish eagle) & $\mathrm{D}$ & M & III, VI, VII & $\mathrm{R}$ & & II \\
\hline Milvus migrants (Black kite) & $\mathrm{U}$ & M & VI, VII & & & II \\
\hline \multicolumn{7}{|l|}{7 Falconidae } \\
\hline Falco cherrug (Saker falcon) & $\mathrm{D}$ & $\mathrm{W}$ & II, III, V & $\mathrm{R}$ & EN & II \\
\hline Falco pelegrinoides (Barbary falcon) & $\mathrm{O}$ & M & I, III, V & $\mathrm{R}$ & & II \\
\hline Falco peregrines $*$ (Peregrine falcon) & $\mathrm{O}$ & M & I, II, II & & & II \\
\hline Falco subbuteo (Eurasian hobby) & $\mathrm{U}$ & M & II, III, V & $\mathrm{R}$ & & II \\
\hline Falco tinnunculus (Common kestrel) & $\mathrm{O}$ & $\mathrm{R}$ & I, II, III & $\mathrm{R}$ & & II \\
\hline \multicolumn{7}{|l|}{ VII Galliformes } \\
\hline \multicolumn{7}{|l|}{8 Phasianidae } \\
\hline Alectoris chukar (Chukar partridge) & $\mathrm{D}$ & $\mathrm{R}$ & II, III, V & $\mathrm{R}$ & & II \\
\hline Coturnix coturnix* (Common quail) & $\mathrm{O}$ & M & VI & & & \\
\hline Tetraogallus himalayensis (Himalayan snowcock) & $\mathrm{P}$ & $\mathrm{R}$ & VI, VII & $\mathrm{R}$ & & II \\
\hline Tetraogallus tibetanus (Tibetan snowcock) & $\mathrm{P}$ & $\mathrm{R}$ & VI, VII & $\mathrm{R}$ & & \\
\hline \multicolumn{7}{|l|}{ VIII Gruiformes } \\
\hline \multicolumn{7}{|l|}{9 Gruidae } \\
\hline Grus grus (Common crane) & $\mathrm{U}$ & M & IV & $\mathrm{R}$ & VU & I \\
\hline Grus nigricollis (Black-necked crane) & $\mathrm{P}$ & $\mathrm{S}$ & IV & $\mathrm{C}$ & & II \\
\hline Grus virgo (Demoiselle crane) & $\mathrm{D}$ & M & IV & $\mathrm{C}$ & & \\
\hline \multicolumn{7}{|l|}{10 Rallidae } \\
\hline Fulica atra (Eurasian coot) & $\mathrm{O}$ & S & $\mathrm{V}, \mathrm{VI}$ & $\mathrm{C}$ & & \\
\hline \multicolumn{7}{|l|}{ IX Charadriiformes } \\
\hline \multicolumn{7}{|l|}{11 Charadriida } \\
\hline Charadrius alexandrinus (Kentish plover) & $\mathrm{O}$ & S & IV & A & NT & \\
\hline Charadrius dubius (Little ringed plover) & $\mathrm{O}$ & S & IV & $\mathrm{R}$ & & \\
\hline Charadrius mongolus (Lesser sand plover) & $\mathrm{D}$ & $\mathrm{S}$ & IV & $\mathrm{C}$ & & \\
\hline Pluvialis fulva (Pacific golden plover) & $\mathrm{C}$ & M & IV & $\mathrm{R}$ & & \\
\hline Pluvialis squatarola (Grey plover) & $\mathrm{D}$ & M & IV & $\mathrm{R}$ & & \\
\hline Vanellus vanellus (Northern lapwing) & $\mathrm{U}$ & $\mathrm{S}$ & IV & $\mathrm{R}$ & & \\
\hline \multicolumn{7}{|l|}{12 Recurvirostridae } \\
\hline Himantopus himantopus* (Black-winged stilt) & $\mathrm{O}$ & M & IV & A & NT & \\
\hline Recurvirostra avosetta (Pied avocet) & $\mathrm{D}$ & $\mathrm{S}$ & IV & & & \\
\hline \multicolumn{7}{|l|}{13 Scolopacidae } \\
\hline Actitis hypoleucos (Common sandpiper) & $\mathrm{C}$ & $\mathrm{S}$ & IV & $\mathrm{R}$ & NT & \\
\hline Calidris alpina (Dunlin) & $\mathrm{U}$ & M & IV & $\mathrm{R}$ & & \\
\hline Calidris ferruginea (Curlew sandpiper) & $\mathrm{U}$ & M & IV & $\mathrm{R}$ & & \\
\hline Calidris temminckii (Temminck's stint) & $\mathrm{U}$ & M & IV & $\mathrm{R}$ & & \\
\hline Gallinago gallinago (Common snipe) & $\mathrm{U}$ & M & IV & $\mathrm{R}$ & & \\
\hline Philomachus pugnax (Ruff) & $\mathrm{U}$ & M & IV & $\mathrm{R}$ & & \\
\hline Limosa limosa (Black-tailed godwit) & $\mathrm{U}$ & M & IV & $\mathrm{R}$ & & \\
\hline Tringa erythropus (Spotted redshank) & $\mathrm{U}$ & M & IV & $\mathrm{R}$ & & \\
\hline Tringa glareola (Wood sandpiper) & $\mathrm{U}$ & $\mathrm{S}$ & IV & $\mathrm{R}$ & & \\
\hline Tringa nebularia* (Common greenshank) & $\mathrm{U}$ & M & IV & & & \\
\hline Tringa ochropus (Green sandpiper) & $\mathrm{U}$ & M & IV & $\mathrm{R}$ & & \\
\hline Tringa totanus (Common redshank) & $\mathrm{U}$ & $\mathrm{S}$ & IV & $\mathrm{A}$ & & \\
\hline
\end{tabular}




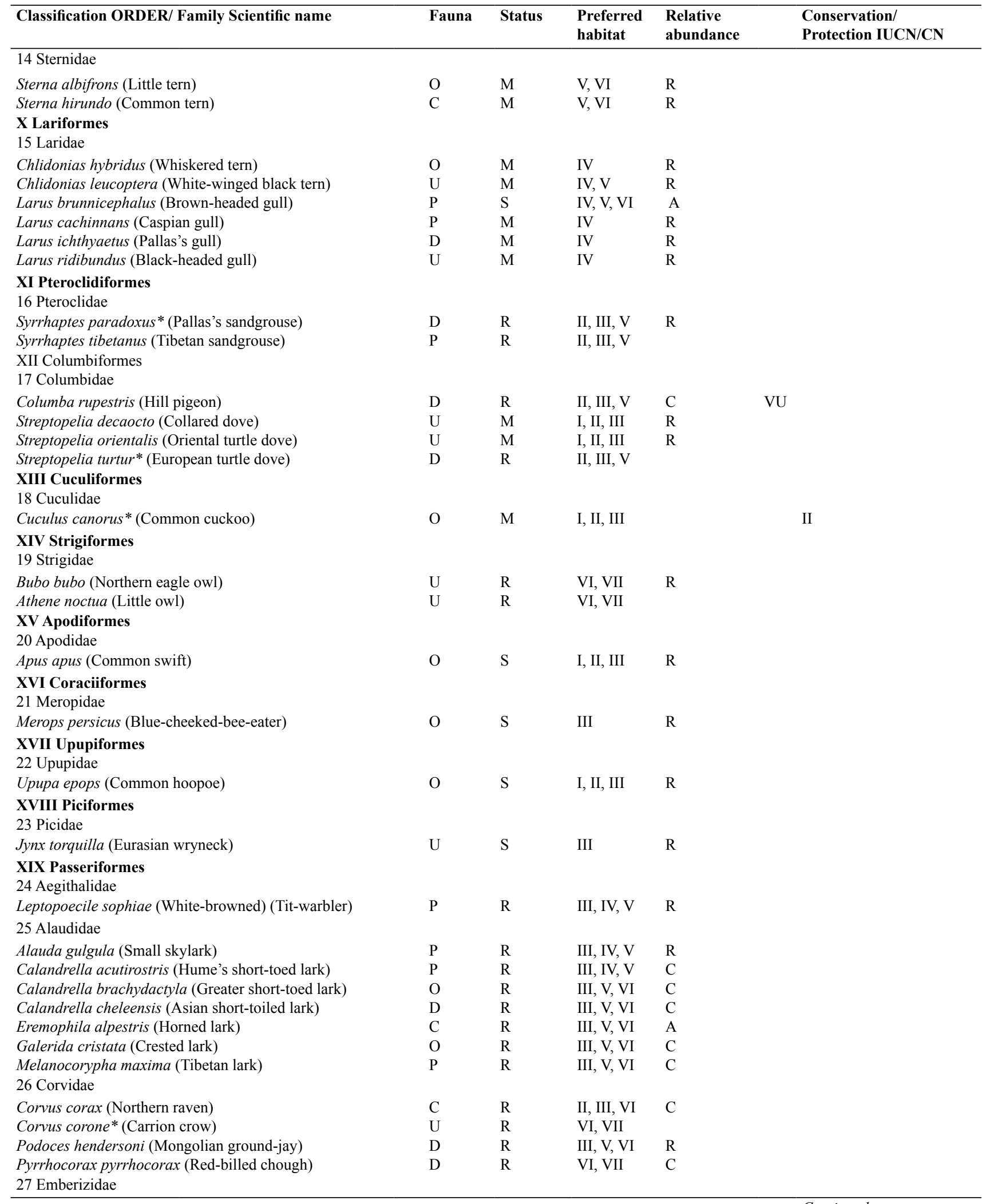




\begin{tabular}{|c|c|c|c|c|c|}
\hline Classification ORDER/ Family Scientific name & Fauna & Status & $\begin{array}{l}\text { Preferred } \\
\text { habitat }\end{array}$ & $\begin{array}{l}\text { Relative } \\
\text { abundance }\end{array}$ & $\begin{array}{l}\text { Conservation/ } \\
\text { Protection IUCN/CN }\end{array}$ \\
\hline Emberiza buchanani (Grey-necked bunting) & $\mathrm{U}$ & M & III, V, VI & $\mathrm{R}$ & \\
\hline Emberiza hortulana* (Ortolan bunting) & $\mathrm{D}$ & M & III, V, VI & & \\
\hline Emberiza pusilla (Little bunting) & $\mathrm{U}$ & $\mathrm{W}$ & IV & $\mathrm{R}$ & \\
\hline $\begin{array}{l}\text { Emberiza Schoeniclus (Common reed bunting) } \\
28 \text { Fringillidae }\end{array}$ & $\mathrm{U}$ & M & IV & $\mathrm{R}$ & \\
\hline Bucanetes mongolicus (Mongolian finch) & $\mathrm{P}$ & $\mathrm{R}$ & I, II, III & $\mathrm{C}$ & \\
\hline Carpodacus erythrinus (Common rosefinch) & $\mathrm{D}$ & M & IV & $\mathrm{R}$ & \\
\hline Carpodacus roborowskii (Tibetan rosefinch) & $\mathrm{U}$ & $\mathrm{R}$ & I, II, III & $\mathrm{R}$ & \\
\hline Carpodacus rubicilla (Great rosefinch) & $\mathrm{P}$ & $\mathrm{R}$ & III, V, VI & $\mathrm{R}$ & \\
\hline Carpodacus sillemi (Sillem's mountain finch) & $\mathrm{D}$ & $\mathrm{R}$ & II, III, VI & $\mathrm{C}$ & DD \\
\hline Fringilla montifringilla (Brambling) & $\mathrm{U}$ & $\mathrm{W}$ & II, V, VI & $\mathrm{C}$ & \\
\hline Leucosticte brandti (Brandt's mountain finch) & $\mathrm{D}$ & $\mathrm{R}$ & III, V, VI & $\mathrm{C}$ & DD \\
\hline Leucosticte nemoricola (Plain mountain finch) & $\mathrm{P}$ & $\mathrm{R}$ & II, V, VI & $\mathrm{R}$ & \\
\hline Linaria cannabina (Common linnet) & $\mathrm{P}$ & $\mathrm{R}$ & III, VI, VI & $\mathrm{R}$ & \\
\hline $\begin{array}{l}\text { Linaria flavirostris (Twite) } \\
29 \text { Hirundinidae }\end{array}$ & $\mathrm{P}$ & $\mathrm{R}$ & I, II, VI & $\mathrm{R}$ & \\
\hline Cecropis daurica (Red-rumped swallow) & $\mathrm{O}$ & M & I, II, III & $\mathrm{R}$ & \\
\hline Hirundo rustica (Barn swallow) & $\mathrm{C}$ & $\mathrm{S}$ & I, II, III & $\mathrm{C}$ & \\
\hline Ptyonoprogne rupestris (Eurasian crag martin) & $\mathrm{D}$ & $\mathrm{S}$ & I, II, III & $\mathrm{C}$ & \\
\hline $\begin{array}{l}\text { Riparia riparia (Sand martin) } \\
30 \text { Laniidae }\end{array}$ & $\mathrm{C}$ & $\mathrm{S}$ & I, II, III & $\mathrm{R}$ & \\
\hline Lanius isabellinus (Red-tailed shrike) & $\mathrm{D}$ & $\mathrm{S}$ & III, V, VI & $\mathrm{R}$ & \\
\hline $\begin{array}{l}\text { Lanius schach (Long-tailed shrike) } \\
31 \text { Motacillidae }\end{array}$ & $\mathrm{W}$ & $\mathrm{S}$ & I, II, III & $\mathrm{C}$ & \\
\hline Anthus richardi (Richard's pipit) & $\mathrm{U}$ & M & IV & $\mathrm{R}$ & \\
\hline Anthus trivialis (Tree pipit) & $\mathrm{U}$ & M & IV & $\mathrm{R}$ & \\
\hline Motacilla alba (White wagtail) & $\mathrm{O}$ & $\mathrm{S}$ & II, III, IV & $\mathrm{C}$ & \\
\hline Motacilla cinerea (Grey wagtail) & $\mathrm{O}$ & M & VI & $\mathrm{R}$ & \\
\hline Motacilla citreola (Citrine wagtail) & $\mathrm{U}$ & $\mathrm{S}$ & IV & $\mathrm{R}$ & \\
\hline $\begin{array}{l}\text { Motacilla flava (Western yellow wagtail) } \\
32 \text { Muscicapidae }\end{array}$ & $\mathrm{U}$ & M & IV & $\mathrm{R}$ & \\
\hline Ficedula albicilla (Taiga flycatcher) & $\mathrm{U}$ & M & I, II, III & $\mathrm{R}$ & \\
\hline Luscinia pectoralis (White-tailed rubythroat) & $\mathrm{H}$ & M & II, III, & $\mathrm{R}$ & \\
\hline Luscinia svecica* (Bluethroat) & $\mathrm{U}$ & M & II, III, V & & \\
\hline Monticola saxatilis (Common rock thush) & $\mathrm{D}$ & M & I, II, III & $\mathrm{R}$ & \\
\hline Oenanthe deserti (Desert wheatear) & $\mathrm{D}$ & $\mathrm{R}$ & II, III, V & $\mathrm{C}$ & \\
\hline Oenanthe oenanthe* (Northern wheatear) & $\mathrm{U}$ & M & III, V, VI & & \\
\hline Oenanthe pleschanka (Pied wheatear) & $\mathrm{D}$ & $\mathrm{S}$ & III, V, VI & $\mathrm{R}$ & \\
\hline Phoenicurus erythrogastrus (Guldenstadt's redstart) & $\mathrm{P}$ & $\mathrm{S}$ & I, II, VII & $\mathrm{C}$ & \\
\hline Phoenicurus ochruros (Black redstart) & $\mathrm{D}$ & $\mathrm{S}$ & III, V, VI & $\mathrm{R}$ & \\
\hline Saxicola torquata* (African stonechat) & $\mathrm{U}$ & M & I, II, III & & \\
\hline $\begin{array}{l}\text { Tarsiger cyanurus (Red-flanked bluetail) } \\
33 \text { Paridae }\end{array}$ & M & M & I, II, III & $\mathrm{R}$ & \\
\hline Pseudopodoces humilis (Ground tit) & $\mathrm{P}$ & $\mathrm{R}$ & I, II, III & $\mathrm{R}$ & \\
\hline $\begin{array}{l}34 \text { Panuridae } \\
\text { Panurus biarmicus (Bearded reedling) } \\
35 \text { Passeridae }\end{array}$ & $\mathrm{P}$ & $\mathrm{R}$ & III, V, VI & & \\
\hline Montifringilla adamsi* (Tibetan snowfinch) & $\mathrm{P}$ & $\mathrm{R}$ & III, V, VI & & \\
\hline Montifringilla nivalis* (White-winged snowfinch) & $\mathrm{P}$ & $\mathrm{R}$ & III, V, VI & & \\
\hline Onychostruthus taczanowskii (White-rumped snowfinch) & $\mathrm{P}$ & $\mathrm{R}$ & II, III, V & $\mathrm{C}$ & \\
\hline Passer domesticus (House sparrow) & $\mathrm{O}$ & $\mathrm{R}$ & I, II, III & $\mathrm{R}$ & \\
\hline Passer montanus (Tree sparrow) & $\mathrm{D}$ & $\mathrm{R}$ & I, II, III & $\mathrm{C}$ & \\
\hline Petronia petronia (Rock sparrow) & $\mathrm{P}$ & $\mathrm{R}$ & I, II, III & $\mathrm{R}$ & \\
\hline Pyrgilauda blanfordi (Blanford's snowfinch) & $\mathrm{U}$ & $\mathrm{R}$ & I, II, III & $\mathrm{R}$ & \\
\hline $\begin{array}{l}\text { Pyrgilauda ruficollis (Rufous-necked snowfinch) } \\
36 \text { Phylloscopidae }\end{array}$ & $\mathrm{P}$ & $\mathrm{R}$ & III, V, VI & $\mathrm{C}$ & \\
\hline Phylloscopus collybita (Chiff-chaff) & $\mathrm{U}$ & M & III, V, VI & $\mathrm{R}$ & \\
\hline Phylloscopus coronatus (Eastern crowned warbler) & M & M & II, III, V & $\mathrm{R}$ & \\
\hline Phylloscopus griseolus* (Sulphur-bellied warbler) & $\mathrm{P}$ & $\mathrm{S}$ & VI, VII & & \\
\hline Phylloscopus humei (Hume's leaf warbler) & $\mathrm{D}$ & $\mathrm{M}$ & III, V, VI & $\mathrm{R}$ & \\
\hline
\end{tabular}




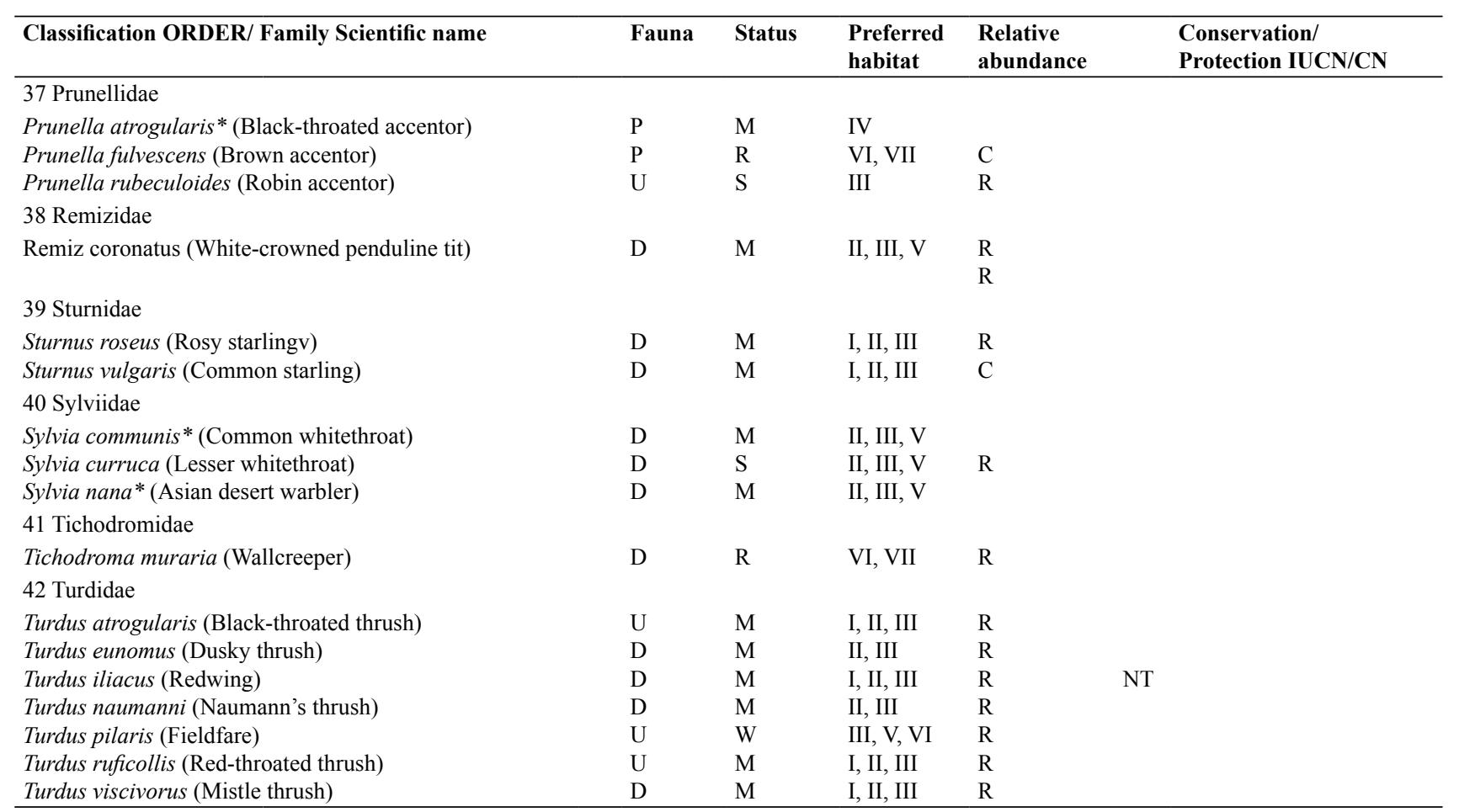

Fauna (distribution pattern): C, Holarictic pattern; O, Circumboreal pattern; U, Palaearctic (Ancient Northern) pattern; D, Central Asian pattern; P-Plateau (Highland) pattern, W-Oriental pattern; H, Mnt. Himalaya-Hengduan pattern; M, Northeastern pattern. Status (residential type): S, summer visitor; W, winter visitor; R, resident; M, migrant (passing migrant). Preferred habitat (Study sites): I, At Athkan; II, Kara Qokka; III, Kara Dong; IV, Ixak Patti; V, Ayak Kum; VI, Aqqik Kul; VII, Whale Lake; Relative abundance (relative proportion of the number of individuals of the species that were observed at all 15x7 sampling events): A, Abundant ( $>1 \%)$; C, Common (0.01-1\%); R, Rare $(<0.01 \%)$. Conservation status: (CN-China), I, Category I Key National Protected Species; II, Category II Key National Protected Species; VU, Vulnerable; NT, Near Threatened; EN, Endangered; DD, Data Deficient (https:// www.iucnredlist.org/, https://commons.wikimedia.org/wiki/Main_Page). *, Based on literature reviews.

\section{CONCLUSION}

The Arjin Mountain Nature Reserve provides protection to a unique assemblage of bird species which may play a critical role in the sustenance of the vulnerable mountain ecosystem. In spite of conservation efforts, however, the bird species are still threatened and have become increasingly vulnerable because of hunting and habitat alteration as human populations in nearby areas have increased and as improved access and increased wealth generally have allowed incursions. In addition to traditional hunting and over-grazing, this area has also witnessed a continuing influx of iron and asbestos miners from the adjacent provinces moving into the reserve. Consequently, rare and endangered bird species, such as raptors, are now absent or scattered in different areas as a result of heavy human disturbances. However, over the last decade, government intervention to control the miners and progressive wildlife protection policy measures have resulted in a significant recovery, especially of raptor populations, for which densities were reported to be very low in the past, as they were illegally hunted by local people for sport and trading. Our surveys suggest that the real conservation issue for birds at present is related to intensifying human-wildlife conflicts resulting in increasing human presence and movements in key wildlife areas along with increasing livestock numbers. We recommend that threats such as hunting, over-grazing, road construction and mining be closely monitored to avoid degradation of protected bird species populations and their natural habitats.

\section{ACKNOWLEDGEMENTS}

This work was financially supported by the Environmental Protection Agency of China, and has been accomplished thanks to the assistance of all the staff of the Arjinshan National Nature reserve. We also thank Kawsar Aman and Qughlukh Mardan for their attention during our research.

\section{Statement of conflict of interest}

The authors have declared is no conflict of interests. 


\section{REFERENCES}

Ablimit, A., 2004. The national-level protected wildlife in Xinjiang (in Chinese). Xinjiang, China.

Achuff, P., and Petocz, R., 1988. Preliminary resource inventory of the Arjin Mountains Nature Reserve, Xinjiang, People's Republic of China: WWF Project 3642 Report, World Wide Fund for Nature, Gland, pp. 78.

Butler, J., Achuff, P. and Jonston, J., 1986. Arjin mountains nature reserve, Xinjiang, People's Republic of China. WWF-IUCN, Gland.

Chen, G., 1985. China establishes more nature reserves. Biol. Conserv., 36: 1-5. (in Chinese)

$\mathrm{Cu}$, P., Xu, H., Ding, H., Cao, M. and Chen, L., 2013. Status quo, problem and countermeasures of bird monitoring in China. J. Ecol. Rural Environ., 29: 403-408. (in Chinese)

Feng, Z., 1991. On the status and conservation of wildlife resources in the Karakorum-Kunlun mountain region, China. Chinese J. Arid Land Res., 4: 65-74. (in Chinese)

Gao, X., 1987. Bird species in eastern Kunlun and Arjin Mountain. Arid Zone Res., 4: 1-10. (in Chinese)

Huang, R. and Gao, X., 1989. Research on the feeding habits of the bird species in Arjin Mountain and its neighboring regions. Sichuan J. Zool., 8: 34-36. (in Chinese)

Jiang, Z., 2020. Insights on the legislation, law enforcement and management of zoonosis from the epidemic of new coronavirus pneumonia
(COVID-19). Biodivers. Sci., 2: 256-261. (in Chinese)

Ma, M., Munkhtsog, B., Xu, F., Turghan, M., Yin S. and Wei, S.D., 2005. Markings as indicator of snow leopard in field survey, in Xinjiang. Chinese J. Zool., 4: 34-39. (in Chinese)

Ma, M., Hu, B., Mei, Y. and Tomas, M., 2010. Survey on bird species and analysis on bird diversity in the Central Kunlun Mountains in the early winter. Arid Zone Res., 27: 230-235. (in Chinese) https://doi. org/10.3724/SP.J.1148.2010.00230

Mardan, T., M., Ma, M., Xu, F. and Yan, W., 2011. Status of snow leopard Uncia uncia and its conservation in the Tumor Peak Natural Reserve in Xinjiang, China. Int. J. Biodiv. Conserv., 3: 497-500.

Mardan, T., Ma, M., Zhang, X. and Zhang, T., 2013. Current population and conservation status of the Tibetan wild ass (Equus kiang) in the Arjin Mountain Natural Reserve, China. Pakistan J. Zool., 45: 12491255.

Qian, Y., Zhang, J. and Zhen, B., 1965. Terrestrial vertebrates of southern Xinjiang. Science Press, Beijing.

Schaller, G., 1998. Wildlife of the Tibetan Steppe. The University of Chicago Press, Chicago.

Zheng, Z., 1976. Zoogeography of China. Science Press, Beijing.

Zhou, Y. and Cheng, Y., 1985. A brief introduction on the geographical distribution of terrestrial vertebrates of eastern Kunlun and Arjin mountain. J. Xinjiang Agric. Univ., 8: 1-10. 